\title{
EVALUACIÓN DE LA PRESENCIA DE LAS MICOTOXINAS ZEARALENONA Y AFLATOXINA TOTAL EN ARROZ SIN CÁSCARA EN LAS PROVINCIAS DE MAYOR PRODUCCIÓN DE ECUADOR
}

\author{
EVALUATION OF THE PRESENCE OF ZEARALENONE AND TOTAL \\ AFLATOXIN MYCOTOXINS IN HUSKED RICE FROM THE HIGHEST \\ PRODUCTIVE PROVINCES OF ECUADOR
}

\section{Andrea Martínez¹, Patricia Garrido', Juan Bravo', Michelle Guijarro", Paulette Andrade'2, Carla Moreno², Israel Vaca², Larry Rivera², Rommel Bentancourt ${ }^{2}$, Paul Vargas ${ }^{3}$, Armando Echeverría ${ }^{4}$ \& Luis Ramos ${ }^{1 *}$}

Recibido: 18 de diciembre 2020 / Aceptado: 10 de junio 2021

DOI 10.26807/ia.v9i2.206

\begin{abstract}
Palabras clave: aflatoxinas, arroz, ELISA competitivo, micotoxinas, zearalenona.
\end{abstract}

Keywords: aflatoxins, rice, ELISA competitive, mycotoxins, zearalenone.

1 Universidad UTE, Facultad de Ciencias de la Ingeniería e Industrias, Centro de Investigación de Alimentos_CIAL, Quito, Ecuador. (andymartinez12@hotmail.com, patricia.garrido@ute.edu.ec, juan. bravo@ute.edu.ec, mguijarro@ute.edu.ec, *correspondencia: luis.ramos@ute.edu.ec)

2 Agencia de Regulación y Control Fito y Zoosanitario_AGROCALIDAD, Coordinación General de Laboratorios, Quito, Ecuador. (paulette.andrade@agrocalidad.gob.ec, carla.moreno@agrocalidad. gob.ec, israel.vaca@agrocalidad.gob.ec, larry.rivera@agrocalidad.gob.ec, rommel.betancourt@agrocalidad.gob.ec)

3 Escuela Politécnica Nacional, Facultad de Ingeniería Química y Agroindustria, Departamento de Ciencias Nucleares, Quito, Ecuador. (paul.vargas@epn.edu.ec)

4 Universidad Internacional del Ecuador, Facultad de Ciencias de la Seguridad y Gestión de Riesgos, Quito, Ecuador. (consultor1@uide.edu.ec) 


\section{RESUMEN}

El arroz es un cultivo de importancia en Ecuador y uno de los cereales más consumidos en este país. Sin embargo, bajo ciertas condiciones, puede contaminarse y contener toxinas exógenas como las micotoxinas. En este sentido, la presente investigación se realizó con la finalidad de levantar información sobre la presencia de las micotoxinas zearalenona y aflatoxina total en arroz sin cáscara, mediante el uso del método inmunológico ELISA, debido a que estos contaminantes comprometen la inocuidad de los alimentos y representan un riesgo para la salud pública. Para ello, se tomaron 41 muestras de los cantones con mayor superficie sembrada de arroz del Ecuador y se analizaron con el método de inmunoensayo ELISA competitivo. Los resultados revelaron la presencia de zearalenona en rangos de concentración entre 1-20 $\mu \mathrm{g} / \mathrm{kg}$ para el $34 \%$ de las muestras y de 41-60 $\mu \mathrm{g} / \mathrm{kg}$ para el $2 \%$ de las muestras; por otro lado, no se detectó la presencia de aflatoxina total en las muestras recolectadas. Se concluye que se detectó la presencia de la micotoxina zearalenona en varias muestras de arroz, pero ventajosamente todas las muestras positivas arrojaron valores por debajo del límite máximo establecido por la Unión Europea (Reglamento (CE) ํㅜ 1881/2006 de la Comisión) de $100 \mu \mathrm{g} / \mathrm{kg}$ para zearalenona y de $4 \mu \mathrm{g} / \mathrm{kg}$ para aflatoxina total.

\section{ABSTRACT}

Rice is an important crop in Ecuador and one of the most consumed cereals in this country. However, under certain conditions, rice can be contaminated and contain exogenous toxins such as mycotoxins. In this sense, the present investigation was carried out to collect information on the presence of the mycotoxins zearalenone and total aflatoxin in husked rice by immunology ELISA method, because the occurrence of these contaminants compromises food safety and represents a risk to public health. In this regard, 41 samples from the cantons with the largest planted area of rice in Ecuador were collected and analyzed by mean of a competitive ELISA immunoassay method. The results revealed the occurrence of zearalenone in concentration ranges between $1-20 \mu \mathrm{g} / \mathrm{kg}$ for $34 \%$ of 
the samples and $41-60 \mu \mathrm{g} / \mathrm{kg}$ for $2 \%$ of the samples; on the other hand, total aflatoxin was not detected in the collected samples. In conclusion, the presence of the mycotoxin zearalenone was detected in several samples of husked rice, however, all samples complied with the maximum limits established by the European Union (Commission Regulation (EC) No 1881/2006) of $100 \mu \mathrm{g} / \mathrm{kg}$ for zearalenone and $4 \mu \mathrm{g} / \mathrm{kg}$ for total aflatoxin.

\section{INTRODUCCIÓN}

La agricultura constituye una de las actividades primarias de mayor importancia en la economía ecuatoriana, debido a la privilegiada ubicación geográfica de este país, que permite producir una amplia gama de productos permanentes y estacionales. Los cultivos estacionales se caracterizan por un ciclo de crecimiento menor a un año, incluso puede ser de pocos meses, y que posterior a la cosecha se inicia un nuevo ciclo con la siembra. En Ecuador, estos cultivos representan el 15,76\% de la superficie de labor agropecuaria, entre estos destacan los cultivos estacionales de mayor producción: arroz, maíz duro seco y papa (INEC, 2016).

El arroz es considerado uno de los cereales más consumidos en el mundo (Gadal et al., 2019). En el ámbito ecuatoriano, el consumo per cápita ha incrementado desde el año 2000, pasando de $42 \mathrm{~kg}$ por persona a 53,2 kg por persona en el año 2013. Por tanto, el arroz ha llegado a constituirse como el principal componente de la canasta básica y un producto esencial dentro de la dieta diaria de los ecuatorianos (MAGAP, 2013). La disponibilidad del producto en el país se basa en la producción interna de arroz, y predominan variedades desarrolladas por el Instituto Nacional de Investigaciones Agropecuarias-INIAP. Se reportó una producción de 1534537 t en el año 2016, con la mayor producción en la región costa con 99,55 \% del total, mientras que en las regiones oriental y sierra los porcentajes fueron 0,26 y $0,19 \%$, respectivamente (INEC, 2016). 
Por otro lado, la producción de arroz en Ecuador se ha visto afectada por algunos problemas fitosanitarios tales como: la presencia de ciertos insectos, por ejemplo, la Sogata (Tagosodesorizicolus) que es el vector del virus de la hoja blanca; la mosquilla o mosca (Hydrellia spp.) que ataca al cultivo en la etapa inicial de su crecimiento (almácigo), y el gusano rojo (Chironomidae) que afecta a las raíces de la planta (Nakandakari, 2017). El principal nematodo fitoparásito que ataca los cultivos de arroz y que ocasiona la descomposición de la raíz es Meloidogyne graminícola (Pérez et al., 2018). En Ecuador se presentan problemas por hongos en arroz; entre los que destacan organismos del género Rhizoctonia que ocasionan las enfermedades del tizón y la pudrición de la vaina en la planta de arroz (Vivas e Intriago, 2012).

Asimismo, también existen problemas asociados a microorganismos alterantes, los cuales pueden contaminar los granos de arroz durante las etapas de postcosecha, almacenamiento, transporte y el procesado. La contaminación por hongos es una problemática mundial, ya que éstos se desarrollan en los cereales cuando existen las condiciones favorables de temperatura y humedad; Lee y Ryu (2016) estimaron que entre el 29 y 61 $\%$ de los cereales sin procesar pueden estar contaminados con una o varias micotoxinas provenientes de ciertas especies de hongos filamentosos.

La Organización de las Naciones Unidas para la Alimentación y la Agricultura (FAO, por sus siglas en inglés) ha determinado que más del 25 $\%$ de los alimentos en el mundo presenta un cierto grado de contaminación con micotoxinas (FAO, 2004) e incluso esta cifra podría estar muy subestimada según estudios recientes (Eskola et al., 2020).

Las micotoxinas son metabolitos secundarios producidos por hongos de distintos géneros y especies; necesarias para el crecimiento del hongo en cultivo puro, y por tal razón, se pueden formar al final de la fase exponencial o al inicio de la fase estacionaria de su desarrollo (Peraica et al., 2000; Duarte y Villamil, 2006). La producción de micotoxinas depende de la especie de hongo, así como del alimento y las condiciones ambientales. Por ejemplo, algunas especies 
de Aspergillus spp. producen aflatoxinas en cereales, maíz, arroz, frutos secos, semillas, legumbres, pasas y vino, mientras que algunas especies de Fusarium spp. son productoras de tricotecenos, fumonisinas y zearalenona en cereales (García, 2008).

Uno de los puntos críticos a considerar sobre la problemática de la contaminación por micotoxinas en el arroz es el almacenamiento, ya que, si no existen los controles adecuados, se generan las condiciones ideales para el desarrollo de los hongos productores de micotoxinas (Troestch y Vega, 2019). Aspergillus flavus, la especie más relacionada con la producción de aflatoxina requiere de determinadas condiciones para su crecimiento y generación de aflatoxinas. En el arroz, este hongo puede crecer a una temperatura entre $6^{\circ} \mathrm{C}$ y $45{ }^{\circ} \mathrm{C}$ con un óptimo a $37{ }^{\circ} \mathrm{C}$ y una actividad de agua $\left(\mathrm{a}_{\mathrm{W}}\right)$ de 0,82 (Parra et al., 2018; Tai et al., 2020). Por tales razones, Troestch y Vega (2019) recomiendan utilizar silos industrializados para tener un monitoreo cuidadoso de la temperatura y humedad del grano almacenado y así disminuir el riesgo de proliferación de los hongos productores de micotoxinas. Sin embargo, en lugares donde el almacenamiento del arroz ocurre de una forma artesanal, no existe un control apropiado de la temperatura y humedad de este alimento, e incluso los riesgos de contaminación por este hongo aumentan cuando los granos entran en contacto con el suelo.

Las micotoxinas mencionadas anteriormente se pueden presentar con mucha frecuencia en alimentos de consumo diario y la generación de estos contaminantes es mayor en zonas con climas cálidos y húmedos, en donde se producen mayoritariamente arroz (Solis, 2018). Además, existen reportes de que las micotoxinas son causantes de afectaciones fisiológicas en humanos, inclusive se las relaciona con el cáncer, por ejemplo, a la zearealenona se ha vinculado con el cáncer de mama (Claeys et al., 2020) y, por lo tanto, las poblaciones cuya dieta consta fundamentalmente de cereales y granos, están en alto riesgo de exposición a alimentos contaminados con micotoxinas y de presentar problemas de salud.

Debido a esta alta probabilidad de contaminación de alimentos por mi- 
cotoxinas, es importante realizar monitoreos continuos de los productos para prevenir principalmente la exposición de los consumidores a estos contaminantes. En la actualidad, existen diversas técnicas analíticas, siendo comunes la aplicación de métodos tipo cribado o screening y confirmatorios.

La técnica ELISA (del inglés "EnzymeLinked ImmunoSorbent Assay") es un análisis que se emplea para la determinación rutinaria de múltiples analitos incluyendo una gran variedad de micotoxinas en diversas matrices (Nolan et al., 2019). Además, esta técnica presenta varias ventajas como el bajo costo de equipos lectores de ELISA, la rapidez de los ensayos, facilidad de operación, sensibilidad analítica y la capacidad de procesamiento de un elevado número de muestras en corto tiempo, con respecto a otras técnicas tales como cromatografía de fluorescencia con derivatización y la cromatografía acoplada a espectrometría de masas (García, 2016).
Los ensayos ELISA presentan variantes como los directos, indirectos, "sandwich", competitivos entre otros. El ensayo ELISA competitivo es el más utilizado para la cuantificación de moléculas de bajo peso molecular como las micotoxinas (antígeno); en este método el antígeno de la muestra y el antígeno fijado en una placa compiten por un anticuerpo específico. Estas interacciones posteriormente se evidencian espectrofotométricamente y se define si una muestra es positiva (sin o poco color) o negativa (coloreada) a la micotoxina, e incluso permite su cuantificación aproximada (Jordan, 2005).

En este contexto, el objetivo principal de esta investigación fue el de evaluar la presencia de las micotoxinas en muestras de arroz sin cáscara de las zonas de mayor producción de Ecuador mediante el ensayo inmunológico ELISA competitivo. 


\section{MATERIALES Y MÉTODOS}

\section{Recolección de las muestras}

Con base a datos del Instituto Nacional de Estadística y Censos (INEC), la toma de las muestras se realizó en los cantones con mayor producción de arroz en las provincias de Los Ríos, Manabí y Guayas. Un total de 41 muestras, con un peso de $1 \mathrm{~kg}$ cada una, se obtuvieron de los silos de cada piladora mediante el protocolo establecido por la Agencia de Regulación y Control Fito y Zoosanitario de Ecuador, (AGROCALIDAD), el cual se basa en los requisitos de la norma NTE INEN-ISO 24333 referente a la toma de muestras de cereales y productos derivados (INEN, 2014). Las muestras se recolectaron y empacaron en fundas plásticas de polietileno con cierre hermético, se colocaron en un recipiente aislante con geles refrigerantes, y se enviaron a las instalaciones de los laboratorios de AGROCALIDAD ubicado en Tumbaco-Quito para su posterior procesamiento y determinación de aflatoxinas totales y zearalenona mediante ensayos ELISA.

\section{Preparación de las muestras}

$5 \mathrm{~g}$ de arroz fueron molidos y colocados en un tubo de centrífuga. Se añadieron $25 \mathrm{~mL}$ de metanol al $70 \%$ $(\mathrm{v} / \mathrm{v})$, el tubo se agitó por $20 \mathrm{~min}$ y luego se centrifugó (Eppendorf, modelo 5804R) a 4000 rpm durante 10 min. Se realizó una dilución de $1 \mathrm{~mL}$ del sobrenadante con $1 \mathrm{~mL}$ de agua destilada, se mezcló por agitación durante 1 min y luego se usaron 50 $\mu \mathrm{L}$ de esta mezcla para el procedimiento de inmunoensayo enzimático competitivo ELISA (BIOO Scientific, 2015).

\section{Procedimiento para el inmunoen- sayo enzimático competitivo ELISA.}

Para la determinación semicuantitativa de zearalenona se utilizó el kit comercial ELISA BiooScientific de zearalenona (ZON) con número de referencia 1035-01 y se siguió el procedimiento detallado en el manual del kit (BIOO Scientific, 2015). Los parámetros de desempeño reportados del método son: recuperación > $80 \%$ y sensibilidad de 0,1 ng/g (ppb). 
La curva de calibración se construyó con los estándares de concentraciones 0,$1 ; 0,25 ; 0,5 ; 1,5$ y $4,5 \mathrm{ng} / \mathrm{mL}, y$ además se preparó un control negativo sin la presencia del estándar. Se colocaron $50 \mu \mathrm{L}$ de cada estándar en orden creciente en cada pocillo, por duplicado. Luego, se colocaron $50 \mu \mathrm{L}$ de los sobrenadantes diluidos de cada muestra de arroz, y se añadieron $100 \mu \mathrm{L}$ del mix de anticuerpos para zearalenona. Las mezclas se agitaron por $1 \mathrm{~min}$. Los pocillos se incubaron durante $30 \min$ a $25{ }^{\circ} \mathrm{C}$ en oscuridad, y, luego las soluciones de los pocillos se desecharon. Los pociIlos se lavaron 3 veces con $300 \mu \mathrm{L}$ de solución de lavado $1 \mathrm{X}$, posteriormente se agregaron $100 \mu \mathrm{L}$ de sustrato TMB y se llevó a incubación por 15 min a $25^{\circ} \mathrm{C}$.

Después se adicionaron $100 \mu \mathrm{L}$ de la solución amortiguadora stop para detener la reacción enzimática. Los pocillos de la placa fueron medidos en un lector de ELISA a una longitud de onda de $450 \mathrm{~nm}$.

Para la determinación semicuantitativa de aflatoxina total, se utilizó el kit comercial ELISA BiooScientific de aflatoxina total con número de referencia 1030-02 y se siguió el proce- dimiento detallado en el manual del kit (BIOO Scientific, 2016). Los parámetros de desempeño reportados del método son: recuperación > 80 \% y sensibilidad de 0,05 ng/g.

La curva de calibración se construyó con los estándares de concentraciones 0,$02 ; 0,06 ; 0,2 ; 0,6$ y $1,5 \mathrm{ng} / \mathrm{mL}$ y además se preparó un control negativo sin la presencia del estándar. Se colocaron $50 \mu \mathrm{L}$ de cada estándar en orden creciente en cada pocillo, por duplicado. Luego, se colocaron $50 \mu \mathrm{L}$ de los sobrenadantes diluidos de cada muestra de arroz y se añadieron $100 \mu \mathrm{L}$ de la solución de anticuerpos para aflatoxinas número 1 . Las mezclas se agitaron por $1 \mathrm{~min}$. Los pocillos se incubaron durante 30 min a $25^{\circ} \mathrm{C}$ y luego las soluciones de los pocillos se desecharon. Se lavaron 3 veces con $250 \mu \mathrm{L}$ de solución de lavado $1 \mathrm{X}$, y posteriormente se agregaron $150 \mu \mathrm{L}$ de la solución de anticuerpos para aflatoxinas número $21 \mathrm{X}$ y los materiales se incubaron durante 30 min a $25^{\circ} \mathrm{C}$ en oscuridad. Se desecharon las soluciones de los pocillos, a continuación, se lavaron 3 veces con $250 \mu \mathrm{L}$ de solución de lavado $1 \mathrm{X} .100 \mu \mathrm{L}$ de sustrato TMB se agregaron a cada pocillo y se mezcló suavemente el contenido de los po- 
cillos por un minuto mientras se incubaba a $25^{\circ} \mathrm{C}$. Después de $15 \mathrm{~min}$ se adicionaron $100 \mu \mathrm{L}$ de la solución amortiguadora stop para detener la reacción enzimática. Los pocillos de la placa fueron medidos en un lector de ELISA a una longitud de onda de $450 \mathrm{~nm}$.

El método ELISA utilizado, corresponde a un protocolo estandarizado o método normalizado (BIOO Scientific, 2015). Adicionalmente, como medida de verificación de desempeño en las condiciones de las mediciones se alternaron muestras de control positivo por duplicado y se determinó con estas el error relativo. En el caso de la zearalenona se utili- zaron como controles los estándares de $1,5 \mathrm{ng} / \mathrm{mL}$ y $4,5 \mathrm{ng} / \mathrm{mL}$, y para aflatoxina total los estándares de 0,6 $\mathrm{ng} / \mathrm{mL}$ y $1,5 \mathrm{ng} / \mathrm{mL}$. El error relativo (ER) se determinó mediante la ecuación (1) a partir de los valores mencionados anteriormente como las concentraciones teóricas (Cteórica) y los resultados obtenidos después del análisis como los valores experimentales (Cexp). Así mismo, la precisión del método se verificó mediante el análisis de réplicas de controles y muestras y se calculó el coeficiente de variación (\% CV) promedio del método.

Error relativo $=\frac{\left(C_{\text {exp }}-C_{\text {teórica }}\right)}{C_{\text {teórica }}} \times 100$ (1)

\section{RESULTADOS}

Se obtuvieron las siguientes curvas de calibración para la cuantificación de zearalenona: $y=-0,2214 \ln (x)+0,4954$ con un $r=0,977$ y $y=-0,1461 \ln (x)+$ 0,2639 con un $r=0,997$ para aflatoxina total. Los controles de zearalelona de 1,5 y $4,5 \mathrm{ng} / \mathrm{mL}$ presentaron resultados experimentales de 1,8 y $5,35 \mathrm{ng} / \mathrm{mL}$, lo que corresponde a ER de 19,9 y $18,9 \%$ respectivamente. Para el caso de aflatoxina total los controles de 0,6 y $1,5 \mathrm{ng} / \mathrm{mL}$ presentaron resultados experimentales de 0,69 y $1,74 \mathrm{ng} / \mathrm{mL}$ lo que corresponde a ER de 14,3 y 16,3\% respectivamente. El CV promedio de las evaluaciones fue de 1,19\%, valor que no supera el $10 \%$ establecido como límite de control del método. Los resultados positivos a micotoxinas en las muestras de arroz sin cáscara se muestran en la Tabla 1. 


\section{Tabla 1. Resultados positivos a zearalenona en muestras de arroz sin cáscara}

\begin{tabular}{lcc}
\hline Provincia & Piladora & $\begin{array}{c}\text { Zearalenona } \\
(\mu \mathrm{g} / \mathrm{kg}) \pm \mathrm{s}\end{array}$ \\
\hline \multirow{6}{*}{ Guayas } & PIL17 & $3,20 \pm 0,04$ \\
& PIL18 & $2,14 \pm 0,04$ \\
& PIL19 & $2,46 \pm 0,01$ \\
& PIL20 & $2,28 \pm 0,03$ \\
& PIL21 & $2,61 \pm 0,03$ \\
& PIL22 & $3,34 \pm 0,06$ \\
& PIL23 & $2,83 \pm 0,01$ \\
& PIL24 & $2,78 \pm 0,03$ \\
& PIL25 & $2,20 \pm 0,00$ \\
& PIL26 & $2,54 \pm 0,04$ \\
Los Ríos & PIL27 & $3,32 \pm 0,04$ \\
\hline Manabí & PIL33 & $9,91 \pm 0,03$ \\
& PIL36 & $51,82 \pm 0,04$ \\
\hline
\end{tabular}

\section{DISCUSIÓN}

Las curvas de calibración obtenidas para la cuantificación de las micotoxinas evaluadas presentaron una adecuada relación entre la concentración de los estándares y los valores de absorbancia relativa, reflejado por altos coeficientes de correlación ( $r$ ) de 0,977 y 0,997 para la zearalenona y aflatoxina total, respectivamente. Estos resultados son similares a los reportados por otros autores (Fajardo et al., 2006; Valderrama et al., 2009;
Omar et al. 2020), y demuestran el buen desempeño del método aplicado. En la evaluación del error relativo con controles positivos se obtuvieron valores menores al $20 \%$, lo que indica también un buen desempeño (veracidad) del método, esto con la consideración de que se trata de un método semi cuantitativo.

En la Tabla 1 se puede apreciar que las concentraciones de zearalenona 
se encuentran entre 1 a $20 \mu \mathrm{g} / \mathrm{kg}$ y 41 a $60 \mu \mathrm{g} / \mathrm{kg}$ para el $34 \%$ y $2 \%$ del total de las muestras, respectivamente. Consecuentemente el $64 \%$ restante de las muestras no evidenciaron la presencia de esta micotoxina.

Debido a que la concentración máxima de zearelonona permitida en arroz no se encuentra establecida en la normativa nacional (INEN, AGROCALIDAD, Ministerio de Salud), se comparó con el umbral de $100 \mu \mathrm{g} / \mathrm{kg}$ establecido por la Comisión de las Comunidades Europeas (2006) en el Reglamento (CE) №1881 para cereales no elaborados distintos al maíz. De acuerdo con esto, el $100 \%$ de las muestras analizadas cumplen y se encuentran bajo el límite establecido por el mencionado Reglamento. Las muestras con mayor concentración de zearalenona pertenecían a la provincia de Los Ríos, donde un valor de $51,8 \mu \mathrm{g} / \mathrm{kg}$ fue determinado en una muestra proveniente del cantón Vinces. Por otra parte, muestras procedentes de cantones como Nobol, Yaguachi, Santa Lucía y Guayaquil pertenecientes a la provincia del Guayas reflejaron concentraciones entre $2,14 \mu \mathrm{g} / \mathrm{kg}$ y $3,34 \mu \mathrm{g} / \mathrm{kg}$. En el caso de la provincia de Manabí, muestras procedentes de los canto- nes Rocafuerte y Sucre exhibieron concentraciones de $2,19 \mu \mathrm{g} / \mathrm{kg}$ y 2,45 $\mu \mathrm{g} / \mathrm{kg}$, respectivamente. Adicionalmente, hay que considerar que es recomendable realizar ensayos confirmatorios de los resultados positivos obtenidos con los métodos de cribado como el ELISA, por ejemplo usando Cromatografía Líquida acoplada a Detector de Espectrometría de Masas, debido a la posibilidad de falsos positivos o variaciones en las concentraciones obtenidas. En este caso, todas las muestras resultaron dentro de los umbrales tomados como referencia, y no fue indispensable su reevaluación por métodos confirmatorios (Okuma et al., 2018). Los resultados obtenidos indican que las muestras de arroz sin cáscara cumplen con la normativa de la UE, sin embargo, es evidente, la presencia de zearalenona en este alimento. Esto podría deberse a las condiciones ambientales favorables para el crecimiento de hongos de las zonas muestreadas, como son la elevada humedad (70 a $80 \%$ ) y temperatura (20-30 $\left.{ }^{\circ} \mathrm{C}\right)$. Además, el Codex Alimentarius indica que los granos pequeños y arrugados pueden presentar más zearalenona que los granos sanos normales (CODEX, 2019). 
Por otro lado, no se detectó la presencia de aflatoxina total en el $100 \%$ de las muestras provenientes de los distintos cantones de las provincias de Los Ríos, Guayas y Manabí. La concentración máxima permitida de aflatoxina total es de $4 \mu \mathrm{g} / \mathrm{kg}$ en todos los cereales y todos los productos de cereales, según lo establecido en el Reglamento (CE) N¹881 de 2006 (Comisión de las Comunidades Europeas). Con estos resultados, se puede inferir que en los cantones evaluados se llevan a cabo buenas prácticas agrícolas (BPA) y buenas prácticas de fabricación (BPF), lo que ayuda a prevenir la generación de estos metabolitos, tal como es establecido por el CODEX (CODEX, 2015). Algunos estudios han demostrado que los nutrientes y el tipo de sustrato son importantes para que exista la producción de aflatoxinas; son favorables altos niveles de carbohidratos y bajos niveles de proteínas, como es el caso del maíz (Morris, 2011). Se sabe que el arroz es uno de los cereales que tiene el mayor contenido de aminoácidos (Tattibayeva, 2017), aspecto que implica un alto contenido de nitrógeno con relación al contenido de carbohidratos. Tal aspecto podría considerarse desfavorable para el crecimiento de los hongos generadores de las aflatoxinas totales.

Estudios anteriores han reportado resultados similares a los de este estudio. Por ejemplo, Morris (2011) obtuvo concentraciones en niveles de riesgo bajo en 46 muestras de arroz provenientes de cinco departamentos de Colombia. Asimismo, Díaz et al. (2001) reportaron la presencia de aflatoxinas con rangos de concentración entre 1 y 13,6 $\mu \mathrm{g} / \mathrm{kg}$ en cada 4 muestras analizadas de un total de 40 muestras de arroz.

\section{CONCLUSIÓN}

Los resultados indicaron que todas las muestras de las provincias de Los Ríos, Manabí y Guayas cumplen con la normativa de la Unión Europea respecto a la máxima concentración permitida de $100 \mu \mathrm{g} / \mathrm{kg}$ para zearale- nona y $4 \mu \mathrm{g} / \mathrm{kg}$ para aflatoxina total en cereales. Sin embargo, hay que considerar que un $36 \%$ de las muestras de arroz presentaron valores positivos (ELISA) para zearalenona en un rango de 1 a $60 \mu \mathrm{g} / \mathrm{kg}$. Este ha- 
llazgo indica que es necesario realizar nuevas evaluaciones e incluir pruebas confirmatorias en el caso de valores superiores a los límites máximos permitidos, por ejemplo, mediante métodos basados en cromatografía líquida acoplada a detector de espectrometría de masas. También es indispensable monitorear y man- tener las buenas prácticas agrícolas en la producción de este alimento de alto consumo en el país, esto debido a que se conoce que las micotoxinas pueden causar graves afectaciones a la salud humana e incluso se las ha vinculado al desarrollo de varios tipos de cáncer.

\section{LISTA DE REFERENCIAS}

BIOO Scientific (2015). Zearalenone (ZON) ELISA: Test Kit Manual. BIOO Food \& Feed Safety.

BIOO Scientific. (2016). Total Aflatoxin ELISA: Test Kit Manual. BIOO Food \& Feed Safety.

Claeys, L., Romano, C., De Ruyck, K., Wilson, H., Fervers, B., Korenjak, M., Zavadil. J., Gunter, M. J., De Saeger, S., De Boevre, M. y Huybrechts, I. (2020). Mycotoxin exposure and human cancer risk: A systematic review of epidemiological studies. Comprehensive Reviews in Food Science and Food Safety, 19(4), 1449-1464. https://doi.org/10.1111/1541-4337.12567.

CODEX. (2015). Norma general para los contaminantes y las toxinas presentes en los alimentos y piensos: CXS STAN 193. FAO y OMS.

CODEX (2019). Standard for rice: CXS STAN 198. FAO y OMS.

Reglamento № (CE) 1881/2006 [Comisión de las Comunidades Europeas]. Por el que se fija el contenido máximo de determinados contaminantes en los productos alimenticios. 19 de diciembre de 2006. 
Denli, M. y Pérez, J. F. (16-17 de octubre de 2006). Contaminación por micotoxinas en Ios piensos: efectos, tratamiento y prevención. XXII Curso de Especialización. FEDNA, Barcelona, España.

Díaz, G. J., Perilla, N. S. y Rojas, Y. (2001). Occurrence of aflatoxins in selected Colombian foods. Mycotoxin Res., 17(1), 15-20. DOI: 10.1007/BF02946113.

Duarte-Vogel, S. y Villamil-Jiménez, L. C. (2006). Micotoxinas en la salud pública. Revista de Salud Pública, 8(supplement 1), 129-135.

Eskola, M., Kos, G., Elliott, C. T., Hajšlová, J., Mayar, S. y Krska, R. (2020). Worldwide contamination of food-crops with mycotoxins: Validity of the widely cited 'FAO estimate' of 25\%. Critical Reviews in Food Science and Nutrition, 60(16), 27732789, DOI: 10.1080/10408398.2019.1658570

Fajardo, E. M., Delgado, I., Riverón, L., Izquierdo, L., Iglesias, N., Álvarez, E., Perojo, A., Costa, N., Tamayo, Y., Jorge, E., Hernández, B., Díaz, Y., Cruces, A., Gutiérrez, N., Puig, A. C., Mandiarote, A., Martínez, R. y Cardoso, D. (2006). Validación de un ELISA tipo inhibición para cuantificar polisacárido Vi en la vacuna antitifoídica cubana vax-TyViââ. VacciMonitor, 15(2), 5-12.

FAO (2004). Reglamentos a nivel mundial para las micotoxinas en los alimentos y en las raciones en el año 2003. http://www.fao.org/3/a-y5499s.pdf

Gadal, N., Shrestha, J., Poudel, M.N. y Pokharel, B. (2019). A review on production status and growing environments of rice in Nepal and in the world. Archives of Agriculture and Environmental Science, 4 (1), 83-87.

García, C. (2008). Análisis de aflatoxinas y ocratoxina A en alimentos y evaluación de la ingesta poblacional [Tesis PhD, Universitat de Valencia].

García-Fonseca, S. (2016). Química supramolecular del estado líquido: disolventes nanoestructurados para la extracción de micotoxinas en productos agroalimentarios [Tesis PhD, Universidad de Córdoba] https://helvia.uco.es/xmlui/handle/10396 /13840 
INEC (2016). Superficie, producción y ventas, según región y provincia arroz (en cáscara). https://www.ecuadorencifras.gob.ec/documentos/web-inec/Estadisticas_ agropecuarias/espac/espac-2018/Tabulados\%20ESPAC\%202018.xIsx

Servicio Ecuatoriano de Normalización INEN (2014). NTE INEN-ISO 24333 Cereales y Productos Derivados: Toma de muestras.

Instituto Nacional de Investigaciones Agropecuarias (2006). Incremento del nivel productivo, social y económico del sector arrocero ecuatoriano, a través de la inversión pública para la generación y transferencia de tecnología del INIAP. www.iniap.gob.ec/nsite/images/stories/descargas/proyectos_inversion_iniap/priorizados_senplades/arroz/Proyecto_mef_arroz.doc

Intriago, M., García, B., Peláez, G., Estupiñan, I. y Villao, F. (1991). Principales enfermedades del arroz en el Ecuador y su manejo. Ministerio de agricultura y ganadería. http://ciat-library.ciat.cgiar.org/Articulos_Ciat/2015/SB_191_R5_U583_Vol.3.

Jordan, W. (2005). Enzyme-Linked Immunosorbent Assay. En Walker J.M., Rapley R. (eds) Medical Biomethods Handbook. Humana Press. https://doi.org/10.1385/159259-870-6:419

Lee, H.J. y Ryu, D. (2017) Worldwide Occurrence of Mycotoxins in Cereals and CerealDerived Food Products: Public Health Perspectives of Their Co-occurrence. Journal of Agricultural and Food Chemistry. 65(33), 7034-7051. https://doi.org/10.1021 /acs.jafc. 6 b04847

MAGAP. (2013). Boletín situacional. http://sinagap.agricultura.gob.ec/phocadownloadpap/cultivo/2013/arroz.pdf

Mera Pinargote, J.L. (2017). Determinación de las principales enfermedades del arroz (Oryza Sativa L.) y grado de incidencia en la zona de Montalvo [Tesis de grado, Universidad Técnica de Babahoyo] http://dspace.utb.edu.ec/handle/49000/3307

Morris, L. (2011). Determinación de aflatoxinas en muestras de maíz (Zea mays) y arroz (Oryza sativa) para consumo humano en cinco departamentos de la Costa Caribe Colombiana mediante cromatografía de alta eficiencia durante seis meses en 2011 [Tesis de maestría, Universidad Nacional de Colombia] https://repositorio.unal. edu.co/handle/unal/8318 
Nakandakari, L. (2017). Problemas fitosanitarios en el cultivo de arroz (Oryza sativa L.) [Tesis de grado, Universidad Nacional Agraria La Molina] http://repositorio.lamolina.edu.pe/handle/UNALM/2988

Nolan, P., Auer, S., Spehar, A., Elliott, C. T. y Campbell K. (2019) Current trends in rapid tests for mycotoxins. Food Additives \& Contaminants: Part A, 36(5), 800-814. DOI: 10.1080/19440049.2019.1595171

Okuma, T. A., Huynh, T. P. y Hellberg, R. S. (2018). Use of enzyme-linked immunosorbent assay to screen for aflatoxins, ochratoxin $\mathrm{A}$, and deoxynivalenol in dry pet foods. Mycotoxin Research, 34(1), 69-75.

Omar, S. S., Haddad, M. A. y Parisi, S. (2020) Validation of HPLC and Enzyme-Linked Immunosorbent Assay (ELISA) Techniques for Detection and Quantification of Aflatoxins in Different Food Samples. Foods, 9(5), 661. https://doi.org/10.3390/ foods9050661

Parra Lizarazu, C. D., Quiroga Selaez, G., Giménez Turba, A. y Flores Quisbert, E. (2018). Aflatoxina B1 de Aspergillus spp. generado en arroz, su detección y cuantificación por métodos fluorométricos y HPLC. Revista Boliviana de Química, 35(5), 134145.

Peraica, M., Radic, B., Lucic, A. y Pavlovic, M. (2000). Efectos tóxicos de las micotoxinas en el ser humano. Bulletin of the World Health Organization. 77 (9), 754-766.

Pérez Iglesias, H.I., Rodríguez Delgado, I. y García Batista, R.M. (2018). Principales enfermedades que afectan al cultivo del arroz en Ecuador y alternativas para su control. Revista Científica Agroecosistemas, 6 (1), 16-27.

Solis, J. (2018). Evaluación de biomarcadores de exposición humana a contaminantes ambientales de origen biológico (micotoxinas) en población infantil indígena de la Huasteca Potosina. [Tesis de maestría, Universidad Autónoma de San Luis Potosí] http://ninive.uaslp.mx/xmlui/handle/i/4535

Tai, B., Chang, J., Liu, Y. y Xing, F. (2020) Recent progress of the effect of environmental factors on Aspergillus flavus growth and aflatoxins production on foods. Food Quality and Safety, 4(1), 21-28. https://doi.org/10.1093/fqsafe/fyz040 
Tattibayeva, D. (2017). Presencia de elementos tóxicos y micotoxinas en cereales de Kazajistán [Tesis PhD, Universidad de Santiago de Compostela] https://minerva.usc .es/xmlui/handle/10347/15680

Troestch Ríos, J. y Vega, A. (2019). Control de los niveles de micotoxinas del arroz consumido en Panamá, como parámetro de inocuidad. Revista Plus Economía, 7(1), $32-42$.

Valderrama, S., Pérez, E., Aldama, Y., Costa, L., Quintana, M., Pérez, G. y García, G. (2009). Establecimiento y validación de un ensayo inmunoenzimático tipo ELISA, empleado en el Control de Calidad del Interferón alfa 2b humano recombinante. Vaccimonitor, 18(1), 8-14.

Vivas, L. e Intriago, D. (2012). Guía para el reconocimiento y manejo de las principales enfermedades en el cultivo de arroz en Ecuador. Boletín divulgativo, 426(1), 1-12. 